\title{
Long Non-crossing Configurations in the Plane
}

\author{
Adrian Dumitrescu • Csaba D. Tóth
}

Received: 21 April 2009 / Revised: 7 June 2010 / Accepted: 15 July 2010 /

Published online: 10 August 2010

(C) Springer Science+Business Media, LLC 2010

\begin{abstract}
We revisit some maximization problems for geometric networks design under the non-crossing constraint, first studied by Alon, Rajagopalan and Suri (ACM Symposium on Computational Geometry, 1995). Given a set of $n$ points in the plane in general position (no three points collinear), compute a longest non-crossing configuration composed of straight line segments that is: (a) a matching, (b) a Hamiltonian path, and (c) a spanning tree. We obtain some new results for (b) and (c), as well as for the Hamiltonian cycle problem.

(i) For the longest non-crossing Hamiltonian path problem, we give an approximation algorithm with ratio $\frac{2}{\pi+1} \approx 0.4829$. The previous best ratio, due to Alon et al., was $\frac{1}{\pi} \approx 0.3183$. The ratio of our algorithm is close to $\frac{2}{\pi} \approx 0.6366$ on a relatively broad class of instances: for point sets whose perimeter (or diameter) is much shorter than the maximum length matching. For instance "random" point sets meet the condition with high probability. The algorithm runs in $O\left(n^{7 / 3} \log n\right)$ time.

(ii) For the longest non-crossing spanning tree problem, we give an approximation algorithm with ratio 0.502 which runs in $O(n \log n)$ time. The previous ratio, $1 / 2$, due
\end{abstract}

A preliminary version of this paper appeared in the Proceedings of the 27th International Symposium on Theoretical Aspects of Computer Science (STACS 2010), Nancy, France, March 2010, pp. 299-310.

A. Dumitrescu was supported in part by NSF CAREER grant CCF-0444188. Part of the research by this author was done at Ecole Polytechnique Fédérale de Lausanne.

C.D. Tóth was supported in part by NSERC grant RGPIN 35586. Part of the research by this author was done at Tufts University.

\footnotetext{
A. Dumitrescu $(\bowtie)$

Department of Computer Science, University of Wisconsin, Milwaukee, USA

e-mail: dumitres@uwm.edu

C.D. Tóth

Department of Mathematics and Statistics, University of Calgary, Calgary, AB, Canada

e-mail: cdtoth@ucalgary.ca
} 
to Alon et al., was achieved by a quadratic time algorithm. Along the way, we first re-derive the result of Alon et al. with a faster algorithm and a very simple analysis.

(iii) For the longest non-crossing Hamiltonian cycle problem, we give an approximation algorithm whose ratio is close to $2 / \pi$ on a relatively broad class of instances: for point sets where the product $\langle$ diameter $\times$ convex hull size $\rangle$ is much smaller than the maximum length matching. Again "random" point sets meet the condition with high probability. However, this algorithm does not come with a constant approximation guarantee for all instances. The algorithm runs in $O\left(n^{7 / 3} \log n\right)$ time. No previous approximation results were known for this problem.

\section{Introduction}

Crossing edges in geometric graphs are undesirable in most cases. Many structures studied in computational geometry, in particular those of minimum Euclidean length, are automatically crossing-free. Such examples include minimum spanning trees, minimum length matchings, minimum traveling salesman tours, Voronoi diagrams, and others. The non-crossing property usually follows from the triangle inequality.

Alon et al. [3] have considered the problems of computing (i) the longest noncrossing matching, (ii) the longest non-crossing Hamiltonian path and (iii) the longest non-crossing spanning tree, given $n$ points in the plane. Although they were unable to prove it, they suspected that all these problems are NP-hard. The survey articles by Eppstein [10, pp. 439] and Mitchell [17, pp. 680] list these as open problems in the area of geometric network optimization. The problem of approximating the maximum non-crossing Hamiltonian cycle is also of interest and wide open [5, pp. 338].

Without the non-crossing condition explicitly enforced, the problems of minimizing or maximizing the length of a spanning tree, Hamiltonian cycle or path, perfect matching, triangulation, etc. has a rich history. For minimization problems, the noncrossing property comes usually for free via the triangle inequality. For maximization problems, however, the non-crossing property conflicts directly with the length maximizing objective. Not surprisingly, much less is known about the crossing-free maximization problems.

Related Work The existence of non-crossing Hamiltonian paths and cycles in geometric graphs has been studied in [2,6]. Various Ramsey-type results for noncrossing spanning trees, paths and cycles have been obtained in [14] and [15]. The Euclidean MAX TSP, the problem of computing a longest straight-line tour of a set of points, has been proven NP-hard in dimensions three or higher [12], while its complexity in the Euclidean plane remains open [17]. In contrast, the shortest noncrossing matching and the shortest non-crossing spanning tree are both computable in polynomial time $[10,17]$, as they coincide with the shortest matching and the shortest spanning tree respectively.

Given a set of $n$ points in general position ${ }^{1}$ in the plane, the results of Alon et al. are as follows: (i) A non-crossing matching whose total length is at least

\footnotetext{
${ }^{1}$ Throughout this paper, a set $S$ of points in the plane is said to be in general position if no three points are collinear.
} 
$2 / \pi$ of the longest (possibly crossing) matching can be computed in $O\left(n^{7 / 3} \log n\right)$ time. (ii) A non-crossing Hamiltonian path whose total length is at least $1 / \pi$ of the longest (possibly crossing) Hamiltonian path can be computed in $O\left(n^{7 / 3} \log n\right)$ time. (iii) A non-crossing spanning tree whose total length is at least $n /(2 n-2) \geq 1 / 2$ of the longest (possibly crossing) spanning tree can be computed in $O\left(n^{2}\right)$ time. Their original results mention somewhat higher running times for (i) and (ii), which were based on the best upper bound at that time on the number of halving lines of a set of $n$ points. The running times have been adjusted to reflect the current best upper bound of $O\left(n^{4 / 3}\right)$ on the number of halving lines as established by Dey [7]. Alon et al. mention that their techniques can be applied to achieve constant factor approximations for the longest triangulation, and the longest bounded-degree spanning tree on $n$ points in the plane, although they do not provide explicit bounds.

Definitions and Notations A geometric graph $G$ is a pair $(V, E)$ where $V$ is a finite set of points in general position in the plane, and $E$ is a set of segments (edges) connecting points in $V$. The length of $G$, denoted $L(G)$, is the sum of the Euclidean lengths of all edges in $G$. The graph $G$ is said to be non-crossing if its edges have pairwise disjoint interiors (collinear triples of points are forbidden in order to avoid overlapping collinear edges).

For a point set $S$, let $\operatorname{conv}(S)$ be the convex hull of $S$, and let $P=P(S)$ denote the perimeter of $\operatorname{conv}(S)$. Denote by $D=D(S)$ the diameter of $S$ and write $n=|S|$. Let $M_{\mathrm{OPT}}$ be a maximum (possibly crossing) matching of $S$, and let $M_{\mathrm{OPT}}^{*}$ be a longest non-crossing matching of $S$; observe that for odd $n, M_{\mathrm{OPT}}$ is a nearly perfect matching, with $(n-1) / 2$ edges. Let $H_{\mathrm{OPT}}$ be a maximum (possibly crossing) Hamiltonian path of $S$, and let $H_{\mathrm{OPT}}^{*}$ be a longest non-crossing Hamiltonian path of $S$. Let $T_{\mathrm{OPT}}$ be a maximum (possibly crossing) spanning tree of $S$, and let $T_{\mathrm{OPT}}^{*}$ be a longest noncrossing spanning tree of $S$. Finally, let $Q_{\text {OPT }}$ be a maximum (possibly crossing) Hamiltonian cycle of $S$, and let $Q_{\mathrm{OPT}}^{*}$ be a longest non-crossing Hamiltonian cycle of $S$. The following inequalities are obvious: $L\left(M_{\mathrm{OPT}}\right) \leq L\left(H_{\mathrm{OPT}}\right) \leq L\left(T_{\mathrm{OPT}}\right)$.

Let $S$ be a set of $n$ points in general position in the plane. We represent the direction of a line with the angle $\alpha \in[0, \pi)$ it makes with the $x$-axis. A supporting line $\ell$ of two points of $S$ is called a halving line if there are $\lfloor(n-2) / 2\rfloor$ and $\lceil(n-2) / 2\rceil$ points, respectively, in the two open halfplanes bounded by $\ell$ [16]. A bisecting line $\ell$ of $S$ is any line that partitions the point set evenly, i.e., neither of the two open halfplanes defined by $\ell$ contains more than $n / 2$ points of $S$ [8]. Observe that any halving line of $S$ is also a bisecting line of $S$. Any bisecting line of $S$ yields (perhaps nonuniquely) a bipartition $S=R \cup B$, with $R \cap B=\emptyset,|| R|-| B|| \leq 1$, with $R$ contained in one of the closed halfplanes determined by $\ell$, and $B$ contained in the other. We call $S=R \cup B$ a linearly separable bipartition, or balanced partition of $S$. Observe that for any direction $\alpha \in[0, \pi)$, there is a bisecting line of direction $\alpha$ (c.f. [8, Lemma 4.4]). Two bisecting lines are called equivalent if they can yield the same balanced partition of $S$. It is well known that the number of non-equivalent bisecting lines of a set is of the same order as the number of halving lines of the set, and any balanced bipartition can be obtained from a halving line [8, pp. 67].

For a polygon $P$, let $V(P)$ denote the set of vertices of $P$. For a geometric graph $G=(V, E)$ and a point $q \notin V$ such that $V \cup\{q\}$ is in general position, we say that $q$ 
sees a vertex $v \in V$ if the segment $q v$ does not intersect any edge of $G$. We also say that $q$ sees an edge $e \in E$, if the triangle formed by $v$ and $e$ does not intersect any other edge of $G$.

Our Results In this paper we further refine the results of Alon et al. regarding noncrossing Hamiltonian paths and spanning trees, and obtain a new result for Hamiltonian cycles. Our results are summarized in the following three theorems.

Theorem 1 For a set $S$ of $n$ points in general position in the plane:

(i) One can compute a non-crossing Hamiltonian path $H$ of length $L(H) \geq$ $\frac{2}{\pi+1} L\left(H_{\mathrm{OPT}}\right)$ in $O\left(n^{7 / 3} \log n\right)$ time if $n \geq 31$. In particular, this yields an approximation algorithm with ratio $\frac{2}{\pi+1} \approx 0.4829$ for the longest non-crossing Hamiltonian path problem.

(ii) One can compute a non-crossing Hamiltonian path $H$ of length $L(H) \geq$ $\frac{2}{\pi} L\left(H_{\mathrm{OPT}}\right)-\frac{P}{\pi}$ in $O\left(n^{7 / 3} \log n\right)$ time. In particular, if the point set satisfies the condition $\frac{P}{\pi} \leq \delta L\left(H_{\mathrm{OPT}}\right)$ for some small $\delta>0$, then $L(H) \geq\left(\frac{2}{\pi}-\delta\right) L\left(H_{\mathrm{OPT}}\right)$.

(iii) Alternatively, one can compute a non-crossing Hamiltonian path $H$ of length $L(H) \geq(1-\varepsilon) \frac{2}{\pi} L\left(H_{\mathrm{OPT}}\right)-\frac{P}{\pi}$ in $O(n \log n / \sqrt{\varepsilon})$ time.

Theorem 2 For a set $S$ of $n$ points in general position in the plane: One can compute a non-crossing spanning tree $T$ of length $L(T) \geq 0.502 \cdot L\left(T_{\mathrm{OPT}}\right)$ in $O(n \log n)$ time. In particular, this yields an approximation algorithm with ratio 0.502 for the longest non-crossing spanning tree problem.

Although our improvement in the approximation ratio for spanning trees is very small, it shows that the "barrier" of $1 / 2$ can be broken. Also, while from a practical standpoint the improvement in the running time is the most significant aspect, from a theoretical perspective the improvement in the approximation ratio is the most challenging part of our result.

Theorem 3 For a set $S$ of $n$ points in general position in the plane, with $|\operatorname{conv}(S)|=$ $h$ :

(i) One can compute a non-crossing Hamiltonian cycle $Q$ of length $L(Q) \geq$ $\frac{2}{\pi} L\left(Q_{\mathrm{OPT}}\right)-(2 h-1) \frac{P}{\pi}$ in $O\left(n^{7 / 3} \log n\right)$ time. In particular, if the point set satisfies the condition $(2 h-1) \frac{P}{\pi} \leq \delta L\left(Q_{\mathrm{OPT}}\right)$ for some small $\delta>0$, then $L(Q) \geq\left(\frac{2}{\pi}-\delta\right) L\left(Q_{\mathrm{OPT}}\right)$.

(ii) Alternatively, one can compute a non-crossing Hamiltonian cycle $Q$ of length $L(Q) \geq \frac{2}{\pi} L\left(Q_{\mathrm{OPT}}\right)-(h+1) \frac{P}{\pi}$ in $O\left(n^{3} \log n\right)$ time.

(iii) Alternatively, one can compute a non-crossing Hamiltonian cycle $Q$ of length $L(Q) \geq(1-\varepsilon) \frac{2}{\pi} L\left(Q_{\mathrm{OPT}}\right)-(2 h-1) \frac{P}{\pi}$ in $O(n \log n / \sqrt{\varepsilon})$ time.

In the formulation of Theorem 1, it may be convenient to replace the condition $\frac{P}{\pi} \leq \delta L\left(H_{\mathrm{OPT}}\right)$ by the condition $\frac{P}{\pi} \leq \delta L\left(M_{\mathrm{OPT}}\right)$, as the latter can be tested in polynomial time. Similarly, in the formulation of Theorem 3, it may be convenient to replace the condition $(2 h-1) \frac{P}{\pi} \leq \delta L\left(Q_{\mathrm{OPT}}\right)$ by the condition $(2 h-1) \frac{P}{\pi} \leq \delta L\left(M_{\mathrm{OPT}}\right)$. 
Fig. 1 A non-crossing alternating spanning path

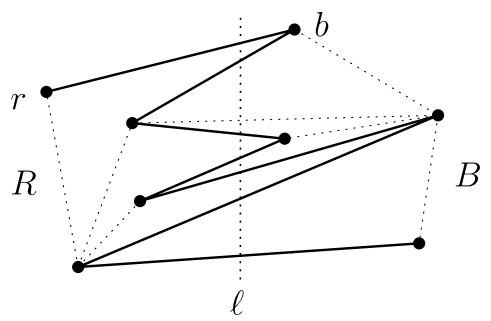

\section{The Hamiltonian Path}

In this section we prove Theorem 1 . Let $S=\left\{p_{1}, \ldots, p_{n}\right\}$. We follow an approach similar to that of Alon et al. using projections and an averaging argument, in conjunction with a result on bipartite embeddings of spanning paths in the plane. Abellanas et al. [1, Theorem 3.1] showed that every linearly separable balanced bipartition $S=R \cup B$ with ||$R|-| B|| \leq 1$, admits an alternating non-crossing spanning path such that the edges cross a separating line $\ell$ at points ordered monotonically along $\ell$, as in Fig. 1. Such a Hamiltonian path can be computed in $O(n \log n)$ time. The alternating path depends only on the bipartition rather than the separating line.

We now recall the algorithm of Abellanas et al. [1]; see Fig. 1 for an example. Let $S=R \cup B$ with ||$R|-| B|| \leq 1$ be the red-blue bipartition given by a vertical line $\ell$ : $R$ on the left, $B$ on the right. Their algorithm constructs an alternating path $A$ in the following way: Let $r b$ be the top red-blue edge of the convex hull conv $(S)$, called the top bridge. If $|R|>|B|$, set $A:=\{r\}$, if $|R|<|B|$, set $A:=\{b\}$, else set $A$ to $\{r\}$ or $\{b\}$ arbitrarily. At every step, recompute the top bridge $r b$ of $S \backslash A$, and add $r$ to $A$ if the last point in $A$ was blue, or add $b$ to $A$ if the last point in $A$ was red. As pointed out by the authors, the resulting path $A$ is non-crossing because $A$ is disjoint from the convex hull of $S \backslash A$ at each step. Note that the constructed path $A$ is not necessarily the longest non-crossing alternating path across $\ell$.

We improve the lower bound of Alon et al. by computing two long paths, and returning the longest of the two. More technical details on this algorithm, A1, will be provided later.

1. Compute a Hamiltonian path, say $H_{1}$, of length at least the perimeter of $\operatorname{conv}(S)$.

2. For each linearly separable bipartition of $S$ (given by a line $\ell$ ), compute a noncrossing alternating Hamiltonian path using [1]. Let $H_{2}$ be the longest Hamiltonian path found in this way.

3. Return the longest of the two paths, $H_{1}$ and $H_{2}$.

Step 2 uses the algorithm of Abellanas et al. [1], but any algorithm that generates a non-crossing alternating path (across the separating line) could be used. The following lemma justifies the first step in our algorithm.

Lemma 1 Given a point set $S$ with $|S|=n \geq 31$, a non-crossing Hamiltonian path $H_{1}$ of length at least $P(S)$ can be computed in $O(n \log n)$ time. The bound on the length is best possible. 

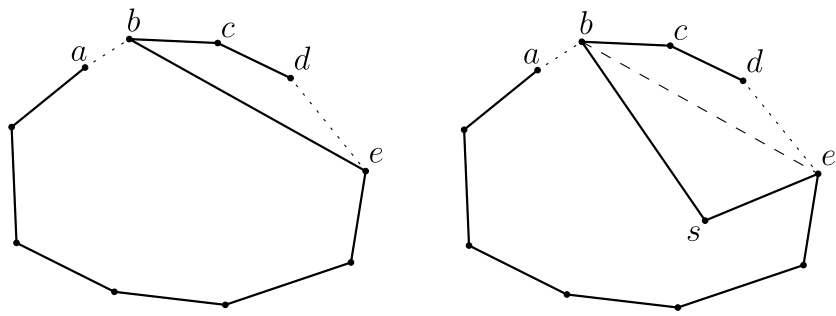

Fig. 2 Constructing a Hamiltonian path of length at least $P(S)$, Case 1. Left: we obtain a Hamiltonian path from $\operatorname{conv}(S)$ by deleting $a b$ and $d e$, and adding be. Right: replacing be with the path (bs, se)

Proof We distinguish two cases based on the number of points in the interior of $\operatorname{conv}(S)$.

Case 1: There is at most one point in the interior of $\operatorname{conv}(S)$. We first construct a Hamiltonian path on the convex hull vertices, and if there is one point in the interior of $\operatorname{conv}(S)$, then we later expand it to a Hamiltonian path of $S$. For the hull vertices, we construct a Hamiltonian path from the convex hull (which is a Hamiltonian cycle) by deleting two edges and adding a diagonal (Fig. 2, left).

Since $S$ contains at least 31 points, there are at least 30 points on the convex hull $\operatorname{conv}(S)$. The sum of (interior) angles of $\operatorname{conv}(S)$ is at least $(30-2) \pi$, hence by averaging, there exist five consecutive hull vertices, whose interior angles sum to at least $\left(5-\frac{1}{3}\right) \pi$. Let $f_{i}, 0 \leq i \leq 5$, be the six hull edges incident to these five consecutive vertices in clockwise order. We may assume, by applying a reflection is necessary, that a shortest edge among these six edges is one of $f_{0}, f_{1}, f_{2}$. Let $a b \in\left\{f_{0}, f_{1}, f_{2}\right\}$ denote a shortest edge, and let $b c, c d$, and $d e$ be the next three edges in clockwise order. For further reference, we have chosen four consecutive edges, $a b, b c, c d$, and $d e$, such that

$$
|a b| \leq \min (|b c|,|c d|,|d e|) \quad \text { and } \quad \angle b c d+\angle c d e \geq\left(2-\frac{1}{3}\right) \pi .
$$

We construct a Hamiltonian path for the hull vertices by deleting edges $a b$ and $d e$ from conv $(S)$, and adding the diagonal be (Fig. 2, left). If there is one point $s \in S$ in the interior of $\operatorname{conv}(S)$, then it sees $b e$, and we expand this path to a Hamiltonian path of $S$ by replacing be with the path ( $b s, s e)$ (Fig. 2, right).

Since $L(\operatorname{conv}(S))=P(S)$, in order to show that we have constructed a Hamiltonian path of length at least $P(S)$, it is enough to show that $|b e| \geq|a b|+|d e|$. Assume that points $b$ and $e$ are fixed, and we vary points $a, c$, and $d$ to maximize $|a b|+|d e|$ subject to the constraints (1). Refer to Fig. 3. Let $p$ be the intersection point of lines $b c$ and $d e$. It follows from (1) that $\angle p c d+\angle p d c \leq \frac{\pi}{3}$, hence $\angle b p e \geq \frac{2 \pi}{3}$, so $p$ lies within the circular segment bounded by the circle $C=\left\{z \in \mathbb{R}^{2}: \angle b z e=\frac{2}{3} \pi\right\}$ (Fig. 3, left). If $|a b|+|d e|$ is maximal, then $p \in C$ (Fig. 3, middle). Since $|a b| \leq \min (|b c|,|c d|)$, for any fixed $p \in C$, the sum $|a b|+|d e|$ is maximal if $|a b|=|b c|=|c d|$. For a fixed $p \in C$, the sum $|a b|+|d e|$ is maximal if $p=d$ and $c$ is the midpoint of $b p$ (Fig. 3, right). We apply the cosine law for the triangle $\triangle b p e$ with $\angle b p e=\frac{2}{3} \pi$ : Letting $|b e|=1, x=|d e|$ and $y=|a b|=|b c|=|c d|$, 

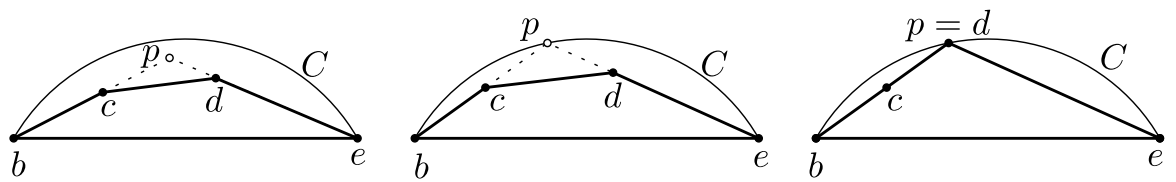

Fig. 3 Left: points $b, c, d$ and $e$, within a circular segment bounded by $C$. Middle: point $p$ is on the circular arc C. Right: $|b c|=|c d|$ and $p=d$

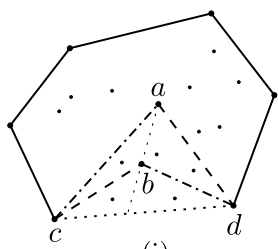

(i)

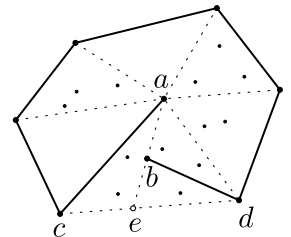

(ii)

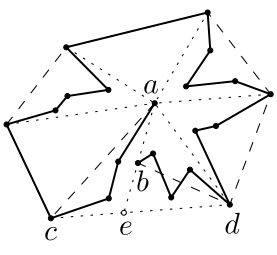

(iii)

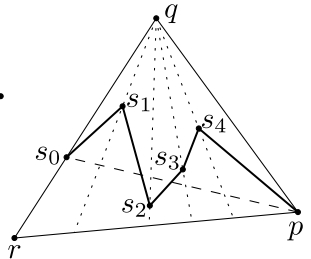

(iv)

Fig. 4 Constructing a path of length at least $P(S)$, Case 2. (i) Points $a, b, c$, and $d$. (ii) The subdivision of the interior of $\operatorname{conv}(S)$. (iii) Extending the Hamiltonian path to other points. (iv) Sweeping triangle $\Delta p q r$ about vertex $q$

we obtain

$$
|b p|^{2}+|e p|^{2}-2|b p| \cdot|e p| \cos \frac{2 \pi}{3}=x^{2}+2 x y+4 y^{2}=1 .
$$

Equivalently, $(x+y)^{2}+3 y^{2}=1$, hence $|a b|+|d e|=x+y \leq 1=|b e|$, with equality for $y=0$.

Case 2: There are at least two points in the interior of $\operatorname{conv}(S)$. In this case, we construct a Hamiltonian path that contains all but one of the edges of $\operatorname{conv}(S)$. Let $a, b \in S$ be two points in the interior of $\operatorname{conv}(S)$. We first construct a Hamiltonian path for all hull vertices and for $a, b$; and then expand it to pass through any other point in the interior of $\operatorname{conv}(S)$. Let $c d$ be a hull edge crossed by the ray $\overrightarrow{a b}$ (Fig. 4(i)); observe that $b \in \Delta a c d$. We construct a non-crossing Hamiltonian path for the hull vertices and $a, b$ by deleting $c d$ from the convex hull and replacing it either with $a c$ and $b d$ or with $a d$ and $b c$. The triangle inequality implies $|c d|<|a c|+|a d|$ and $|c d|<|b c|+|b d|$, and so $|c d|<\max (|a c|+|b d|,|a d|+|b c|)$. That is, with one of the two possible choices, we obtain a Hamiltonian path of length at least $P(S)$.

Suppose that more than two points in $S$ are in the interior of $\operatorname{conv}(S)$. Let $e$ denote the intersection point of segment $c d$ and ray $\overrightarrow{a b}$. Assume without loss of generality that we have added the edges $a c$ and $b d$. Subdivide the interior of $\operatorname{conv}(S)$ into triangles as follows: Connect every hull vertex to $a$, and subdivide $\Delta a c d$ into two triangles, $\Delta a c e$ and $\Delta a d e$ (Fig. 4(ii)). Assign the hull edges of the Hamiltonian path to the adjacent triangle (with one vertex at $a$ ), assign edge $a c$ to $\Delta a c e$, and assign edge $b d$ to $\Delta a d e$. We expand each edge of the current path to a path that passes through all vertices lying in the triangle assigned to that edge (Fig. 4(iii)).

Each triangle $\Delta p q r$ in the subdivision is assigned to an edge $p s_{0}$ connecting a vertex $p$ to a point $s_{0} \in q r$ on the opposite side (Fig. 4(iv)), where $p s_{0}$ may be $p r$. 
Order the points lying in $\Delta p q r$ by a radial sweep about vertex $q$, sweeping from $\overrightarrow{q s_{0}}$ to $\overrightarrow{q p}$. Denoting the points in the interior of $\Delta p q r$ by $s_{1}, s_{2}, \ldots, s_{t}$, for some $t \geq 0$, in this order, we can replace edge $s_{0} p$ by the path $\left(s_{0}, s_{1}, \ldots, s_{t}, p\right)$. This path is non-crossing, it lies entirely in $\Delta p q r$, and its length is at least that of $p s_{0}$.

Lower bound construction. To see that the bound is best possible, consider a point configuration with a diameter pair $a, b$, where $|a b|=1$, and all the other $n-2$ points placed in a small $\varepsilon$-neighborhood of the midpoint of $a b$. Both, the length of the maximum Hamiltonian path (even with crossings allowed), and the perimeter of the convex hull are arbitrarily close to 2 , if $n \geq 4$ and $\varepsilon>0$ is sufficiently small.

We make use of the fact that if $n$ is even then the two endpoints of an alternating path are on opposite sides of the separating line $\ell$. If $n$ is odd, we first construct an alternating path for a specific subset of $n-1$ points, and then augment it to a Hamiltonian path on all $n$ points using the following lemma.

Lemma 2 Let $S=R \cup B$ with ||$R|-| B|| \leq 1$, be a linearly separable bipartition given by line $\ell$. Let $q \in S$, and $A$ be a non-crossing alternating path on $S \backslash\{q\}$ such that its (consecutive) edges cross $\ell$ at points ordered monotonically along $\ell$. Then $q$ sees one edge of $A$. Hence $A$ can be extended to a Hamiltonian path $H$ on $S$, with $L(A)<L(H)$. The path $H$ can be computed in $O(n)$ time.

Proof Compute the visibility polygon of $q$, the boundary of all points visible from $q$ if the path $A$ is opaque. Let $V$ be the set of edges of path $A$ that appear on the boundary of the visibility polygon, that is, the edges that are (partially) visible from $q$. Consider the directed graph $G=(V, E)$, where $(e, f) \in E$ if $e$ blocks the total visibility of $f$ from $q$, that is, there is a point $r \in f$ such that an endpoint of $e$ lies in the relative interior of $q r$. The visibility polygon of $q$ and the graph $G$ can be computed in $O(n)$ time [4, pp. 844], [18, pp. 650]. If $G$ is acyclic, then there is a node of in-degree 0 , that is, an edge $e$ of $A$ partially visible from $q$ and not blocked by any other edge of $A$. Hence, the edge $e$ is entirely visible from $q$. In the rest of the proof, we show that $G$ is acyclic.

Suppose to the contrary that $G$ has a directed cycle $\left(e_{1}, e_{2}, \ldots, e_{m}\right)$ where $e_{i}$ blocks the total visibility of $e_{i+1}$. Refer to Fig. 5. Each edge has a counterclockwise first and second endpoint as viewed from $q$. Since each edge in the cycle blocks another edge, exactly one endpoint of each $e_{i}$ is visible to $q$. If the first (resp., second) endpoint of $e_{i}$ is not visible to $q$, then the same holds for $e_{i+1}$, for $i=1,2, \ldots, m$. Assume, by applying a reflection if necessary, that $q$ sees the first endpoint of each $e_{i}$, but not the second one.

Consider a coordinate system in which the bisecting line $\ell$ is vertical. Assume, by applying a central symmetry about $q$ if necessary, that $q$ lies in the closed halfplane left of $\ell$. Let $p$ denote the point of $A$ visible from $q$ directly above $q$. Assume without loss of generality that $p \in e_{1}$. The counterclockwise first endpoint of $e_{1}$ is its right endpoint. Hence, the portion of $e_{1}$ between its right endpoint and $p$ is visible from $q$. Since $\ell$ crosses $e_{1}$ to the right of $q$, the intersection point $a=\ell \cap e_{1}$ is also visible from $q$. Let segment $a b$ be a maximal continuous portion of $\ell$ visible from $q$ (that is, $a b$ is a component of the intersection of $\ell$ with the visibility polygon of $q$ ). Since the 
Fig. 5 Point $q$ partially sees edges $e_{1}, \ldots, e_{5}$ that cyclically block one another. The visibility polygon of $q$ is shaded gray. The point visible from $q$ and lying directly above $q$ is $p \in e_{1}$. The bisecting line $\ell$ crosses $e_{1}$ at $a$. One component of the intersection of $\ell$ and the visibility polygon of $q$ is segment $a b$, with $b \in e_{k}$

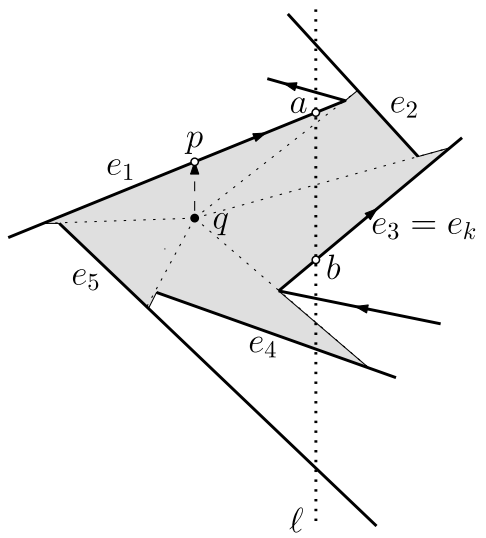

visibility of $q$ is bounded by edges of the cycle in any direction, we have $b \in e_{k}$ for some $1<k \leq m$. The path $A$ cannot intersect $\ell$ between $a=\ell \cap e_{1}$ and $b=\ell \cap e_{k}$, and so $e_{1}$ and $e_{k}$ are consecutive edges of $A$, that is, $e_{1}$ and $e_{k}$ are adjacent.

Note that the lower side of $e_{1}$ and the upper side of $e_{k}$ are visible from $q$. The edge of $A$ incident to the counterclockwise first (i.e., right) endpoint of $e_{1}$ must cross $\ell$ above $a$. Similarly, the counterclockwise first endpoint of $e_{k}$ is its left endpoint and the edge of $A$ incident to it must cross $\ell$ below $b$. Hence the common endpoint of $e_{1}$ and $e_{k}$ can only be the left endpoint of $e_{1}$ and the right endpoint of $e_{k}$. These endpoints, however, are different: they are on opposite sides of $\ell$. We have reached a contradiction, so we conclude that $G$ is acyclic.

Fix a Cartesian coordinate system $\Gamma$. By relabeling the points if necessary, assume that the optimal path is $H_{\mathrm{OPT}}=\left(p_{1}, p_{2}, \ldots, p_{n}\right)$. For two points $p_{i}, p_{j} \in S$, let $\beta_{i, j}$ be the angle in $[0, \pi)$ formed by the line through $p_{i} p_{j}$ and the $x$-axis. If $n$ is odd, then a bisecting line of direction $\alpha$ (for any $\alpha$ ) must be incident to at least one point of $S$, and we denote an arbitrary such point by $q_{\alpha}$.

Algorithm A1 STEP 1. Compute a non-crossing Hamiltonian path $H_{1}$ of length at least $P(S)$, by Lemma 1 .

STEP 2. If $n$ is even, then for all non-equivalent bisections of $S$ (i.e., for all balanced bipartitions of $S$ ), compute a non-crossing alternating path using the algorithm of Abellanas et al. [1], and let the longest such path be $H_{2}$. If $n$ is odd, then for all non-equivalent bisections of $S$, compute a non-crossing alternating path of the even point set $S \backslash\left\{q_{\alpha}\right\}$ using the algorithm of [1] and let the longest such path be A. Augment $A$ with vertex $q_{\alpha}$ by Lemma 2 to a Hamiltonian path $H_{2}$.

STEP 3. Output the longest of the two paths $H_{1}$ and $H_{2}$.

By Lemma 1, the running time of STEP 1 is $O(n \log n)$. Since the number of halving lines of an $n$-element point set is $O\left(n^{4 / 3}\right)$ and all can be generated within this time [7], the running time of STEP 2 is $O\left(n^{7 / 3} \log n\right)$, consequently the total running time of A1 is also $O\left(n^{7 / 3} \log n\right)$. We proceed with the analysis of the approximation ratio. 
Fig. 6 The rotated coordinate system $\Gamma_{\alpha}$ and a non-crossing alternating spanning path $H_{\alpha}=\left(p_{1}, p_{3}, p_{4}, p_{2}\right)$. Notice that $L\left(H_{\alpha}\right) \geq\left|x_{\sigma(1)}\right|+$ $2\left|x_{\sigma(2)}\right|+2\left|x_{\sigma(3)}\right|+\left|x_{\sigma(4)}\right|=$ $\left|x_{1}\right|+2\left|x_{3}\right|+2\left|x_{4}\right|+\left|x_{2}\right|$. A longest spanning path is $H_{\mathrm{OPT}}=\left(p_{1}, p_{2}, p_{3}, p_{4}\right)$

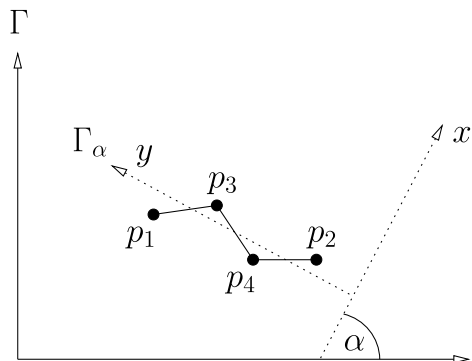

Assume first that $n$ is even. For each $\alpha \in[0, \pi)$, let $\Gamma_{\alpha}$ be a (rotated) coordinate system, obtained from $\Gamma$ via a counterclockwise rotation by $\alpha$, and with the $y$-axis dividing evenly the point set $S$. Let $x_{i}=x_{i}(\alpha)$ be the $x$-coordinate of point $p_{i}$ with respect to $\Gamma_{\alpha}$. Let $H_{\alpha}$ be a non-crossing alternating path with respect to a balanced bipartition induced by the $y$-axis of $\Gamma_{\alpha}$, as computed by the algorithm. See Fig. 6 for a small example.

There are $O(1)$ balanced bipartitions given by any halving line of $S$. Recall that $H_{\alpha}$ does not depend continuously on $\alpha$; it depends only on the discrete bipartition. Assume that $H_{\alpha}=\left(p_{\sigma(1)}, p_{\sigma(2)}, \ldots, p_{\sigma(n)}\right)$, where $\sigma$ is a permutation of $[n]$; here $\sigma$ depends on the bipartition (hence also on $\alpha$ ). Let $W_{\alpha}$ denote the width of $S$ in direction $\alpha$, that is, the width of the smallest parallel strip of direction $\alpha$ that contains $S$. Since $H_{\alpha}$ alternates between the two sides of the $y$-axis of $\Gamma_{\alpha}$, by projecting on the $x$-axis of $\Gamma_{\alpha}$, we get

$$
\begin{aligned}
L\left(H_{\alpha}\right) & \geq\left|x_{\sigma(1)}\right|+2\left|x_{\sigma(2)}\right|+\cdots+2\left|x_{\sigma(n-1)}\right|+\left|x_{\sigma(n)}\right| \\
& =2 \sum_{i=1}^{n}\left|x_{i}\right|-\left|x_{\sigma(1)}\right|-\left|x_{\sigma(n)}\right| \\
& =\sum_{j=1}^{n-1}\left(\left|x_{j}\right|+\left|x_{j+1}\right|\right)+\left|x_{1}\right|+\left|x_{n}\right|-\left|x_{\sigma(1)}\right|-\left|x_{\sigma(n)}\right| \\
& \geq \sum_{j=1}^{n-1}\left(\left|x_{j}\right|+\left|x_{j+1}\right|\right)-W_{\alpha} \\
& \geq \sum_{j=1}^{n-1}\left|p_{j} p_{j+1}\right|\left|\cos \left(\beta_{j, j+1}-\alpha\right)\right|-W_{\alpha} .
\end{aligned}
$$

In the 4th line of the above chain of inequalities, we use the fact that $p_{\sigma(1)}$ and $p_{\sigma(n)}$ lie on opposite sides of the $y$-axis of $\Gamma_{\alpha}$ since $n$ is even. Hence $\left|x_{\sigma(1)}\right|+\left|x_{\sigma(n)}\right| \leq$ $\left|p_{\sigma(1)} p_{\sigma(n)}\right| \leq W_{\alpha}$. In the 5th line, we make use of the following inequality, which is the key property in the approach used by Alon et al. in their approximation algorithm for finding large non-crossing matchings: For any two points $p_{i}, p_{j} \in S$, $\left|p_{i} p_{j}\right|\left|\cos \left(\beta_{i, j}-\alpha\right)\right| \leq\left|x_{i}\right|+\left|x_{j}\right|$, with equality if and only if the two points lie on opposite sides of the $y$-axis of $\Gamma_{\alpha}$. 
Assume now that $n$ is odd. The coordinate system $\Gamma_{\alpha}$ is defined in the same way. For a given $\alpha$, let $H_{\alpha}^{\prime}$ be a non-crossing alternating path with respect to a balanced bipartition of $S \backslash\left\{q_{\alpha}\right\}$, as computed by the algorithm. Let $\sigma$ be the permutation of $[n]$, such that $H_{\alpha}^{\prime}=\left(p_{\sigma(1)}, p_{\sigma(2)}, \ldots, p_{\sigma(n-1)}\right)$ and $p_{\sigma(n)}=q_{\alpha}$. Let $H_{\alpha}$ be the Hamiltonian path obtained from $H_{\alpha}^{\prime}$ by augmenting it with $p_{\sigma(n)}$. Here $x_{\sigma(n)}=0$ since $p_{\sigma(n)}=q_{\alpha}$ is incident to the bisecting line.

$$
\begin{aligned}
L\left(H_{\alpha}\right)>L\left(H_{\alpha}^{\prime}\right) & \geq\left|x_{\sigma(1)}\right|+2\left|x_{\sigma(2)}\right|+\cdots+2\left|x_{\sigma(n-2)}\right|+\left|x_{\sigma(n-1)}\right| \\
& =2 \sum_{i=1}^{n-1}\left|x_{\sigma(i)}\right|-\left|x_{\sigma(1)}\right|-\left|x_{\sigma(n-1)}\right| \\
& =2 \sum_{i=1}^{n}\left|x_{i}\right|-\left|x_{\sigma(1)}\right|-\left|x_{\sigma(n-1)}\right| \\
& \geq \sum_{j=1}^{n-1}\left(\left|x_{j}\right|+\left|x_{j+1}\right|\right)-W_{\alpha} \\
& \geq \sum_{j=1}^{n-1}\left|p_{j} p_{j+1}\right|\left|\cos \left(\beta_{j, j+1}-\alpha\right)\right|-W_{\alpha} .
\end{aligned}
$$

We again have $\left|x_{\sigma(1)}\right|+\left|x_{\sigma(n-1)}\right| \leq W_{\alpha}$ because $n-1$ is even, thus $p_{\sigma(1)}$ and $p_{\sigma(n-1)}$ lie on opposite sides of the bisecting line.

Thus in both cases, $L\left(H_{\alpha}\right) \geq \sum_{j=1}^{n-1}\left|p_{j} p_{j+1}\right|\left|\cos \left(\beta_{j, j+1}-\alpha\right)\right|-W_{\alpha}$. Recall: for even $n, H_{2}$ is the longest of the $O(k)$ Hamiltonian non-crossing paths $H_{\alpha_{i}}$ over all $O(k)$ balanced bipartitions of $S$. (A given angle $\alpha_{i}$ yields $O(1)$ balanced partitions, and corresponding alternating paths denoted here $H_{\alpha_{i}}$.) The situation is similar for odd $n$. We thus also have for each $\alpha \in[0, \pi)$ :

$$
L\left(H_{2}\right) \geq \sum_{j=1}^{n-1}\left|p_{j} p_{j+1}\right|\left|\cos \left(\beta_{j, j+1}-\alpha\right)\right|-W_{\alpha} .
$$

Note that

$$
\int_{0}^{\pi}\left|\cos \left(\beta_{j, j+1}-\alpha\right)\right| \mathrm{d} \alpha=\int_{0}^{\pi}|\cos \alpha| \mathrm{d} \alpha=2,
$$

and according to Cauchy's surface area formula [21], we have $\int_{0}^{\pi} W_{\alpha} \mathrm{d} \alpha=P(S)$. By integrating both sides of the previous inequality over the $\alpha$-interval $[0, \pi]$, we obtain

$$
\begin{aligned}
\pi L\left(H_{2}\right) & \geq 2 \sum_{j=1}^{n-1}\left|p_{j} p_{j+1}\right|-P(S)=2 L\left(H_{\mathrm{OPT}}\right)-P(S), \\
L\left(H_{2}\right) & \geq \frac{2}{\pi} L\left(H_{\mathrm{OPT}}\right)-\frac{P(S)}{\pi} .
\end{aligned}
$$


We now improve the old approximation ratio of $\frac{1}{\pi} \approx 0.3183$ to $\frac{2}{\pi+1} \approx 0.4829$, by balancing the lengths of the two paths computed in STEP 1 and STEP 2. Set $c=\frac{\pi+1}{2}$.

Case 1: $L\left(H_{\mathrm{OPT}}\right) \leq c P(S)$. By considering the path computed in STEP 1, we get a ratio of at least

$$
\frac{L\left(H_{1}\right)}{L\left(H_{\mathrm{OPT}}\right)} \geq \frac{P(S)}{L\left(H_{\mathrm{OPT}}\right)} \geq \frac{P(S)}{c P(S)}=\frac{2}{\pi+1} .
$$

Case 2: $L\left(H_{\mathrm{OPT}}\right) \geq c P(S)$. By considering the path computed in STEP 2 (inequality (4)), we get a ratio of at least

$$
\frac{L\left(H_{2}\right)}{L\left(H_{\mathrm{OPT}}\right)} \geq \frac{\frac{2}{\pi} L\left(H_{\mathrm{OPT}}\right)-\frac{1}{\pi} P(S)}{L\left(H_{\mathrm{OPT}}\right)} \geq \frac{2}{\pi}-\frac{1}{c \pi}=\frac{2}{\pi}\left(1-\frac{1}{\pi+1}\right)=\frac{2}{\pi+1} .
$$

Observe that if the point set satisfies the condition $\frac{P(S)}{\pi} \leq \delta L\left(H_{\mathrm{OPT}}\right)$, then by (4), we have

$$
L(H) \geq \frac{2}{\pi} L\left(H_{\mathrm{OPT}}\right)-\delta L\left(H_{\mathrm{OPT}}\right)=\left(\frac{2}{\pi}-\delta\right) L\left(H_{\mathrm{OPT}}\right) .
$$

This concludes the proofs of parts (i) and (ii) of Theorem 1.

(iii) With the same approach as in [3], a Hamiltonian path of length at least (1 $\varepsilon) \frac{2}{\pi} L\left(H_{\mathrm{OPT}}\right)-\frac{P(S)}{\pi}$ can be found by considering only $b / \sqrt{\varepsilon}$ angles $\theta_{i}=\frac{i \pi \sqrt{\varepsilon}}{b}$, for $i=0,1, \ldots,\lfloor b / \sqrt{\varepsilon}\rfloor$, where $b$ is a suitable absolute constant. The resulting running time is $O(n \log n / \sqrt{\varepsilon})$. This concludes the proof of Theorem 1 .

For an example illustrating part (ii), consider a set of $n$ random points uniformly selected in a convex region, say of unit diameter. Then with high probability $L\left(M_{\mathrm{OPT}}\right)=\Omega(n)$, thus also $L\left(H_{\mathrm{OPT}}\right)=\Omega(n)$. Since $P(S) \leq \pi$, we have $\frac{P(S)}{\pi} \leq \delta L\left(H_{\mathrm{OPT}}\right)$ with high probability, for a very small $\delta=\Theta(1 / n)$. Thus according to inequality (4), the path returned by A1 is a $(2 / \pi-o(1))$ approximation of the optimal path for random point instances.

Remark Lemma 1 applies for $n \geq 31$ only. In the range $7 \leq n \leq 30$, one can use a slightly different balancing argument showing that there is a non-crossing Hamiltonian path of length at least $2 D$, where $D=D(S)$ is the diameter of the point set $S$. Then in inequalities (2) and (3), we can use the diameter $D$ instead of the width $W_{\alpha}$ of $S$ in direction $\alpha$. This approach, however, leads to a smaller approximation ratio, $\frac{4}{3 \pi} \approx 0.4244$.

\section{The Spanning Tree}

In this section we prove Theorem 2. Let $S=\left\{p_{1}, \ldots, p_{n}\right\}$, where $p_{i}=\left(x_{i}, y_{i}\right)$. Given a point $p \in S$, the star centered at $p$, denoted $S_{p}$, is the spanning tree on $S$ whose edges join $p$ to all the other points. Since $S$ is in general position, $S_{p}$ is non-crossing for any $p \in S$. An extended star centered at $p$ is a spanning tree of $S$ consisting of paths of length 1 or 2 (edges) connecting $p$ to all the other points. See Fig. 7. While the star centered at a point is unique, there may be many extended stars centered at 
Fig. 7 A star (left) and a non-crossing extended star (right) on a same point set, both centered at the same point $p$

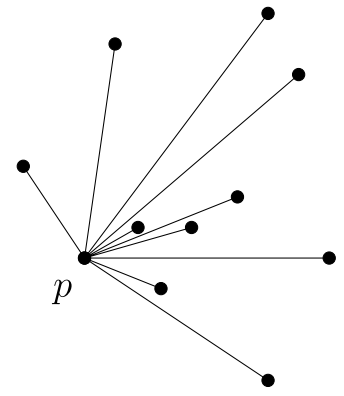

the same point, and some of them may be self-crossing. In particular $S_{p}$ is also an extended star.

The algorithm of Alon et al. computes the $n$ stars centered at each of the points, and then outputs the maximum one. The algorithm takes quadratic time, and the analysis shows a ratio of $\frac{n}{2 n-2}$ (which tends to $1 / 2$ in the limit). Their algorithm works in any metric space. As pointed out by Alon et al., the ratio $1 / 2$ is best possible (in the limit) for this specific algorithm. We first re-establish the $1 / 2$ approximation ratio using a faster algorithm, and also with a simpler analysis. Our algorithm works also in any metric space (however in this general setting, the running time remains quadratic).

Algorithm A2 Compute a diameter of the point set, and output the maximum of the two stars centered at one of its endpoints.

Obviously the algorithm runs in $O(n \log n)$ time, with bottleneck being the diameter computation [19]. Let $a b$ be a diameter pair, and assume without loss of generality that $|a b|=1$. The ratio $1 / 2$ (or even $\frac{n}{2 n-2}$ ) follows from the next lemma in conjunction with the obvious upper bound $L\left(T_{\mathrm{OPT}}\right) \leq n$ (or $\left.L\left(T_{\mathrm{OPT}}\right) \leq n-1\right)$.

Lemma 3 Let $S_{a}$ and $S_{b}$ be the stars centered at the points $a$ and $b$, respectively. Then $L\left(S_{a}\right)+L\left(S_{b}\right) \geq n$.

Proof Assume that $a=p_{1}, b=p_{2}$. For each $i=3, \ldots, n$, the triangle inequality for the triple $a, b, p_{i}$ gives

$$
\left|a p_{i}\right|+\left|b p_{i}\right| \geq|a b|=1 \text {. }
$$

By summing up we have

$$
L\left(S_{a}\right)+L\left(S_{b}\right)=\sum_{i=3}^{n}\left(\left|a p_{i}\right|+\left|b p_{i}\right|\right)+2|a b| \geq(n-2)+2=n .
$$

We now continue with the new algorithm that achieves a (provable) $\frac{1}{2}+\frac{1}{500}$ approximation ratio within the same running time $O(n \log n)$. We suspect that the approximation ratio of A3 is substantially better than that of the quadratic time algorithm of Alon et al., namely $1 / 2$. However at the moment we can only prove a lower bound of 0.502 on this ratio. 

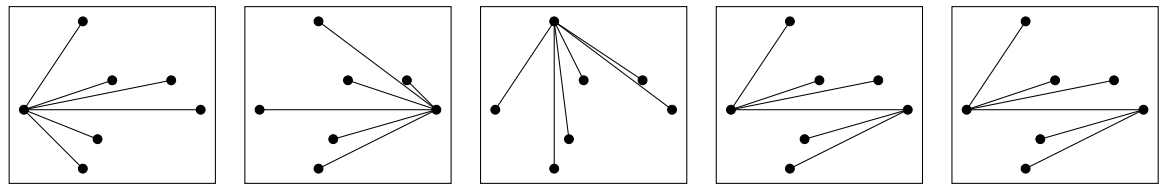

Fig. 8 Three stars $S_{a}, S_{b}, S_{h}$, and two extended stars $E_{a}, E_{b}$

Fig. 9 A diameter pair $a, b$ at unit distance, and the three vertical strips $V_{a}, V_{m}$, and $V_{b}$. The two circular arcs $\gamma_{a}$ and $\gamma_{b}$ of unit radius centered at $a$ and $b$ intersect at the point $(1 / 2, \sqrt{3} / 2)$. All points of $S$ above $a b$ lie in the region bounded by $a b, \gamma_{a}$ and $\gamma_{b}$

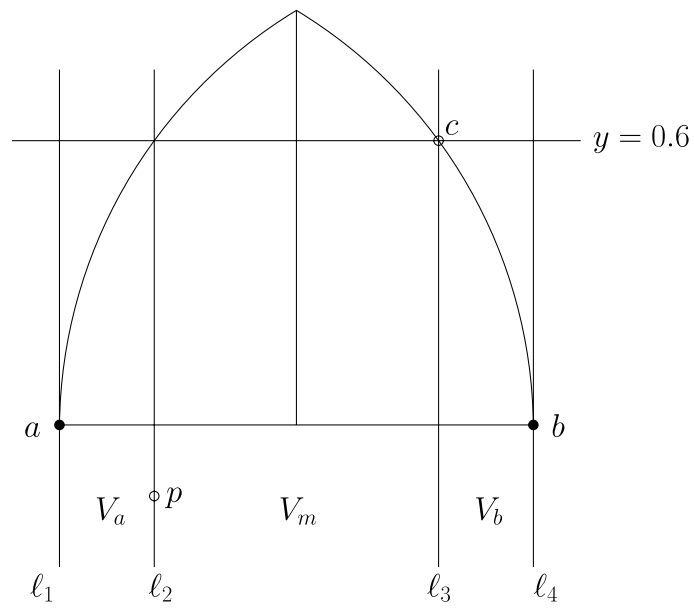

Algorithm A3 Compute a diameter $a b$ of the point set, and output the longest of the 5 non-crossing structures $S_{a}, S_{b}, S_{h}, E_{a}, E_{b}$, described below.

Assume without loss of generality that $a b$ is a horizontal unit segment, where $a=(0,0)$ and $b=(1,0)$. Let $h=\left(x_{h}, y_{h}\right)$ be a point in $S$ with a largest value of $|y|$ (farthest from the $y$-axis). By symmetry, we can assume that $y_{h} \geq 0 . S_{a}, S_{b}$, and $S_{h}$ are the 3 stars centered at $a, b$, and $h$, respectively. $E_{a}$ and $E_{b}$ are two non-crossing extended stars centered at $a$ and $b$, respectively, described below. Each of the five structures can be computed in $O(n \log n)$ time, so the total execution time is also $O(n \log n)$. The five structures for a small example are shown in Fig. 8.

We will use four parameters, which we collect here for easy reference. By the end of the proof we will set

$$
w=0.6, \quad t=0.6, \quad \delta=0.05, \quad \text { and } \quad z=0.48,
$$

in order to maximize the approximation ratio ( $w$ and $t$ are two lengths, while $\delta$ and $z$ are two numbers).

Let $\ell_{1}, \ell_{2}, \ell_{3}$, and $\ell_{4}$, be four parallel vertical lines: $\ell_{1}: x=0, \ell_{2}: x=\frac{1-w}{2}, \ell_{3}$ : $x=\frac{1+w}{2}, \ell_{4}: x=1$; refer to Fig. 9. Obviously, all points in $S$ lie in the strip bounded by $\ell_{1}$ and $\ell_{4}$. Let $V_{m}$ be the vertical parallel strip symmetric about the midpoint of $a b$ and of width $w$. We refer to $V_{m}$ as the middle strip; $V_{m}$ is bounded by the vertical lines $\ell_{2}$ and $\ell_{3}$. Let $V_{a}$ and $V_{b}$ be the two vertical strips of width $\frac{1-w}{2}$ bounded by $\ell_{1}$ and $\ell_{2}$, and by $\ell_{3}$ and $\ell_{4}$, respectively. Let $c=\left(x_{c}, y_{c}\right)$ be the intersection point 
between $\ell_{3}$ and the circular arc $\gamma_{a}$ of unit radius centered at $a$ and sub-tending an angle of $60^{\circ}$. We have $x_{c}=\frac{1+w}{2}$ and

$$
y_{c}=\sqrt{1-\left(\frac{1+w}{2}\right)^{2}}=: t .
$$

We now describe the two extended star structures $E_{a}$ and $E_{b}$. See Fig. 10 for an example. To construct $E_{a}$, connect $a$ with each point in the right strip $V_{b}$. Note that $b \in V_{b}$, thus $V_{b} \neq \varnothing$. Call $S_{a}^{\prime}$ the resulting star. The edges of this star together with the vertical line $\ell_{3}$ divide $V_{a} \cup V_{m}$ into convex regions (wedges with a common apex $a$ ) ordered top-down. The subset of points in each wedge can be computed using binary search in overall $O(n \log n)$ time (over all wedges). $S_{a}^{\prime}$ is extended (augmented) as follows. In the interior of each wedge, say paq, all points are connected to either $a$ or $p$, forming a star, to maximize the total length of the star. Let $\overrightarrow{a p^{\prime}}$ and $\overrightarrow{a p^{\prime \prime}}$, $p^{\prime}, p^{\prime \prime} \in V_{b}$, be the rays of minimum and maximum slope, respectively. Connect all points in $V_{a} \cup V_{m}$ lying below $\overrightarrow{a p^{\prime}}$ to either $a$ or $p^{\prime}$. Similarly, connect all points in $V_{a} \cup V_{m}$ lying above $\overrightarrow{a p^{\prime \prime}}$ to either $a$ or $p^{\prime \prime}$. Denote the resulting extended star structure by $E_{a}$. The construction of $E_{b}$ is analogous. It is clear by construction that both $E_{a}$ and $E_{b}$ are non-crossing.

The next five lemmas provide various upper and lower bounds we need for establishing the approximation ratio of the Algorithm A3.

Lemma 4 For each $p \in S$, let $d_{\max }(p)$ denote the maximum distance between $p$ and other points in $S$. Then

$$
L\left(T_{\mathrm{OPT}}\right) \leq\left[\sum_{i=1}^{n} d_{\max }\left(p_{i}\right)\right]-1
$$

Proof Consider $T_{\mathrm{OPT}}$ rooted at $a$. Let $\pi(v)$ denote the parent of a (non-root) vertex $v$. Uniquely assign each edge $\pi(v) v$ of $T_{\mathrm{OPT}}$ to vertex $v$. Obviously, $L(\pi(v) v) \leq$ $d_{\max }(v)$ holds for each edge in the tree. By adding up the above inequalities, and taking into account that $d_{\max }(a)=|a b|=1$, the lemma follows.

Lemma 5 Assume that $\sum_{i=1}^{n}\left|y_{i}\right| \geq \delta$ for some constant $\delta \in[0,1]$. Then

$$
L\left(S_{a}\right)+L\left(S_{b}\right) \geq 2 n \sqrt{\frac{1}{4}+\delta^{2}} .
$$

Proof Recall that $x_{i} \in[0,1]$ and $y_{i} \in[-\sqrt{3} / 2, \sqrt{3} / 2]$. By the optics reflection principle,

$$
\sqrt{x_{i}^{2}+y_{i}^{2}}+\sqrt{\left(1-x_{i}\right)^{2}+y_{i}^{2}} \geq 2 \sqrt{\frac{1}{4}+y_{i}^{2}} .
$$


Therefore

$$
L\left(S_{a}\right)+L\left(S_{b}\right)=\sum_{i=1}^{n}\left(\sqrt{x_{i}^{2}+y_{i}^{2}}+\sqrt{\left(1-x_{i}\right)^{2}+y_{i}^{2}}\right) \geq 2 \sum_{i=1}^{n} \sqrt{\frac{1}{4}+y_{i}^{2}} .
$$

It can be checked that the function $f(x)=\sqrt{\frac{1}{4}+x^{2}}$ is convex $\left(f^{\prime \prime}(x) \geq 0\right.$ for $x \in$ $[0,1])$, thus by Jensen's inequality we get

$$
\begin{gathered}
2 \sum_{i=1}^{n} \sqrt{\frac{1}{4}+y_{i}^{2}} \geq 2 n \sqrt{\frac{1}{4}+\left(\frac{\sum_{i=1}^{n}\left|y_{i}\right|}{n}\right)^{2}} \geq 2 n \sqrt{\frac{1}{4}+\delta^{2}}, \\
L\left(S_{a}\right)+L\left(S_{b}\right) \geq 2 n \sqrt{\frac{1}{4}+\delta^{2}} .
\end{gathered}
$$

Lemma 6 Let $n_{a}$ and $n_{b}$ denote the number of points in the left and right vertical strips $V_{a}$ and $V_{b}$. Then $L\left(E_{a}\right) \geq \frac{1+w}{4}\left(n+n_{b}\right)$, and similarly $L\left(E_{b}\right) \geq \frac{1+w}{4}\left(n+n_{a}\right)$. Consequently $L\left(E_{a}\right)+L\left(E_{b}\right) \geq \frac{1+w}{4}\left(2 n+n_{a}+n_{b}\right)$. $E_{a}$ and $E_{b}$ can be constructed in $O(n \log n)$ time.

Proof The distance between $\ell_{1}$ and $\ell_{3}$ is $w+\frac{1-w}{2}=\frac{1+w}{2}$. By an argument similar to that in the proof of Lemma 3, the connection cost for a wedge with $m$ points is at least $\frac{1+w}{4} m$. Therefore the total length of $E_{a}$ is

$$
L\left(E_{a}\right) \geq \frac{1+w}{2} n_{b}+\frac{1+w}{4}\left(n-n_{b}\right)=\frac{1+w}{4}\left(n+n_{b}\right) .
$$

The estimation of $L\left(E_{b}\right)$ is analogous. The running time has been established previously.

Lemma 7 Assume that $\sum_{i=1}^{n}\left|y_{i}\right| \leq \delta n$ and $y_{h} \geq t$. Then $L\left(S_{h}\right) \geq(t-\delta) n$.

Proof

$$
L\left(S_{h}\right) \geq \sum_{i=1}^{n}\left(y_{h}-y_{i}\right)=n y_{h}-\sum_{i=1}^{n} y_{i} \geq n y_{h}-\sum_{i=1}^{n}\left|y_{i}\right| \geq n y_{h}-\delta n \geq(t-\delta) n .
$$

Lemma 8 Let $y_{0} \geq 0$. Assume that $\left|y_{h}\right| \leq t$. Let $p \in S$ be a point in the middle strip $V_{m}$, with $y$-coordinate satisfying $|y| \leq y_{0}$. Then $d_{\max }(p) \leq|p c| \leq \sqrt{w^{2}+\left(y_{0}+t\right)^{2}}$.

Proof It is straightforward to check that the maximum distance from such a point is attained for a point $p$ on $\ell_{2}$ with $y$-coordinate $-y_{0}$. The furthest point from $p$ in the allowed region is $c$. Hence

$$
d_{\max }(p) \leq|p c|=\sqrt{w^{2}+\left(y_{0}+t\right)^{2}} .
$$


Approximation Ratio Set $y_{0}=0.15$ and parameters $w, t, \delta$ and $z$ as in (5). By Lemma 8 , if $p$ is a point in the middle strip $V_{m}$, with $y$-coordinate satisfying $|y| \leq y_{0}$, we have $d_{\max }(p) \leq \sqrt{0.6^{2}+0.75^{2}} \leq 0.9605$. We distinguish the following four cases to complete our estimation of the approximation ratio.

Case 1: $\sum_{i=1}^{n}\left|y_{i}\right| \geq \delta n$. The algorithm outputs ${ }^{2} S_{a}$ or $S_{b}$. By Lemma 5, the approximation ratio is at least

$$
\frac{L\left(S_{a}\right)+L\left(S_{b}\right)}{2 L\left(T_{\mathrm{OPT}}\right)} \geq \sqrt{\frac{1}{4}+\delta^{2}} \geq 0.502 .
$$

Case 2: $\sum_{i=1}^{n}\left|y_{i}\right| \leq \delta n$ and $y_{h} \geq t$. The algorithm outputs $S_{h}$. By Lemma 7, the approximation ratio is at least $t-\delta=0.55$.

Case 3: $\sum_{i=1}^{n}\left|y_{i}\right| \leq \delta n$ and $y_{h} \leq t$ and $n_{a}+n_{b} \geq(1-z) n$. The algorithm outputs $E_{a}$ or $E_{b}$. We only need the last inequality in estimating the length. By Lemma 6, the approximation ratio is at least

$$
\frac{L\left(E_{a}\right)+L\left(E_{b}\right)}{2 L\left(T_{\mathrm{OPT}}\right)} \geq \frac{1+w}{4} \cdot \frac{2 n+n_{a}+n_{b}}{2 n} \geq \frac{(1+w)(3-z)}{8}=1.6 \cdot 2.52 / 8=0.504 .
$$

Case 4: $\sum_{i=1}^{n}\left|y_{i}\right| \leq \delta n$ and $y_{h} \leq t$ and $n_{a}+n_{b} \leq(1-z) n$. The algorithm outputs $S_{a}$ or $S_{b}$. There are at least $z n=0.48 n$ points in the middle strip $V_{m}$. Observe that at most $n / 3$ points in $V_{m}$ have $\left|y_{i}\right| \geq 0.15$; otherwise we would have

$$
\sum_{i=1}^{n}\left|y_{i}\right| \geq \sum_{V_{m}}\left|y_{i}\right|>0.15 \cdot \frac{n}{3}=0.05 n=\delta n,
$$

a contradiction. It follows that at least $12 n / 25-n / 3=11 n / 75$ points in the middle strip have $\left|y_{i}\right| \leq 0.15$. By Lemma 4 and Lemma 8 ,

$$
L\left(T_{\mathrm{OPT}}\right) \leq \frac{64 n}{75}+0.9605 \cdot \frac{11 n}{75} \leq 0.9943 n .
$$

The approximation ratio is at least

$$
\frac{L\left(S_{a}\right)+L\left(S_{b}\right)}{2 L\left(T_{\mathrm{OPT}}\right)} \geq \frac{n}{2 \cdot 0.9943 n} \geq 0.502 .
$$

This completes the list of cases and thereby the proof of Theorem 2 .

Remarks Conforming with Theorem 4.1 of [3], or with our Lemma 3, there exists a vertex $v \in S$ such that $L\left(S_{v}\right) \geq L\left(T_{\mathrm{OPT}}\right) / 2$. Alon et al. pointed out that the constant $1 / 2$ in their theorem is best possible in a metric space: take two points $p_{1}$ and $p_{2}$ at distance 1 from each other, and replace $p_{i}$ by $n / 2$ copies in a small neighborhood. We further note here that the constant $1 / 2$ in their theorem is also best possible in the geometric setting, since for the above example, $L\left(T_{\mathrm{OPT}}\right) \geq L\left(H_{\mathrm{OPT}}\right) \approx n-1$,

\footnotetext{
${ }^{2}$ Here and in other instances it is meant that the algorithm outputs a structure at least as long as these.
} 
Fig. 10 The non-crossing structure $E_{a}$ for an example with $n=16$ points on the circle. The middle strip $V_{m}$ is bounded by the two dashed vertical lines

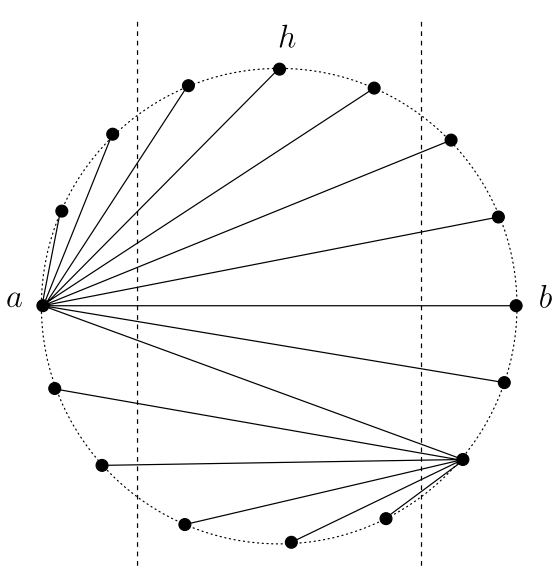

as given by a spanning path that alternates between the two groups of points, and since the length of any star is about $n / 2$. The example in Fig. 10 with $n$ points ( $n$ even) equally spaced along a circle shows that the constant 0.502 measuring the approximation ratio achieved by our Algorithm A3 cannot be improved to anything larger than $\frac{2}{\pi} \approx 0.6366$. Indeed, the lengths of the five structures computed by the algorithm are $L\left(S_{a}\right)=L\left(S_{b}\right)=L\left(S_{h}\right)=L\left(E_{a}\right)=L\left(E_{b}\right)=(1-o(1)) \frac{2}{\pi} n$, while $L\left(T_{\mathrm{OPT}}\right) \geq L\left(H_{\mathrm{OPT}}\right)=(1-o(1)) n$.

\section{The Hamiltonian Cycle}

In this section we present the proof of Theorem 3, which is similar (including notation) to that of Theorem 1 . The rotated coordinate system $\Gamma_{\alpha}$, and the $x$-coordinates $x_{i}$ with respect to this system are denoted in the same way. By relabeling the points if necessary, assume that the optimal cycle is $Q_{\mathrm{OPT}}=\left(p_{1}, p_{2}, \ldots, p_{n}\right)$, with the convention that $p_{n+1}=p_{1}$. We will present two algorithms, $\mathrm{A} 4$ and $\mathrm{A} 4^{\prime}$, fulfilling the requirements in parts (i) and (ii) of the theorem.

We approximate $Q_{\mathrm{OPT}}$ by first constructing a non-crossing alternating path $A$ on a subset of $S$, and then completing it to a non-crossing cycle using convex hull vertices. Let $S=S^{\prime} \cup S^{\prime \prime}$, where $S^{\prime}$ is the set of convex hull vertices and $S^{\prime \prime}$ is the set of interior points. Let $S^{\prime}=\left\{p_{j_{1}}, p_{j_{2}}, \ldots, p_{j_{h}}\right\}$. Put $h=\left|S^{\prime}\right|, m=\left|S^{\prime \prime}\right|$, thus $n=h+m$.

The general plan is as follows. First we construct a non-crossing alternating path $A$ for a bipartition of $S^{\prime \prime}$. If the two endpoints of $A$ lie on $\operatorname{conv}\left(S^{\prime \prime}\right)$, each can be connected to some vertex of $\operatorname{conv}(S)$ and these two vertices can be connected on the hull of $S$, resulting in a non-crossing cycle of $S \backslash Z$, where $Z \subset S^{\prime}$. Then this partial cycle of $S$ can be augmented with the remaining points (in $Z$ ) in a non-crossing fashion, and with no decrease in length.

We need to observe that the alternating path $A$ on the subset $S^{\prime \prime} \subset S$ produced by the algorithm of Abellanas et al. [1] is not good enough for this strategy: even though one endpoint of $A$ (the first computed by the algorithm) is always on the convex hull of $S^{\prime \prime}$, the other endpoint might be blocked by edges of $A$, so that $A$ might not be 
Fig. 11 A non-crossing alternating path of $S^{\prime \prime}$ obtained by the algorithm of Abellanas et al. For the purpose of cycle construction, the path is non-extendible from its second endpoint, vertex 7. Here $S^{\prime}=\{a, b, c, d\}$, $S^{\prime \prime}=\{1,2,3,4,5,6,7\}$, and $S=S^{\prime} \cup S^{\prime \prime}$

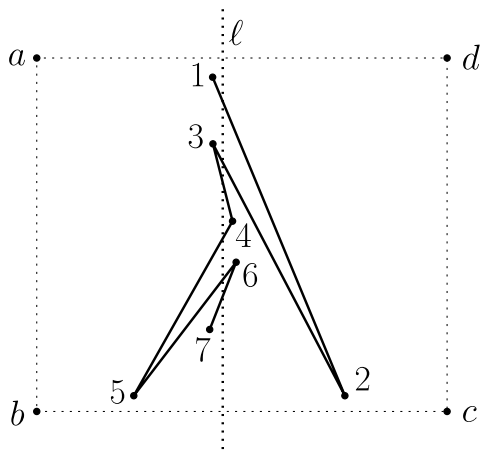

extendible to a non-crossing Hamiltonian cycle (an example is shown in Fig. 11). Here, we give a stronger result (Lemma 9) that fits our purpose (for an even number of points). We then apply Lemma 9 for constructing an alternating path of $S^{\prime \prime}$.

Lemma 9 Let $S$ be an n-element point set. Every linearly separable bipartition $S=R \cup B$, with $|R|=|B|$, admits an alternating non-crossing spanning path such that (1) the edges cross any separating line $\ell$ at points ordered monotonically along $\ell$; and (2) the two endpoints are incident to the two distinct edges of $\operatorname{conv}(S)$ that connect $R$ and $B$ (the two red-blue bridges). Such a Hamiltonian path can be computed in $O(n \log n)$ time. We refer to the underlying procedure as the two-endpoint path construction algorithm.

Proof We modify the algorithm of Abellanas et al. for path construction, so that the path is grown from the two endpoints and the two sub-paths merge "in the middle". See also Fig. 12. Recall that $S=R \cup B$, and $|R|=|B|$, thus $|S|$ is even. Let $r_{1} b_{1}$ and $r_{2} b_{2}$ be the top and bottom red-blue edges of the convex hull conv $(S)$, respectively, called top and bottom bridges; it is possible that $r_{1}=r_{2}$ or $b_{1}=b_{2}$ but not both (for $n \geq 3$ ). One endpoint is an endpoint of the top bridge, and the other endpoint is an endpoint of the bottom bridge, and they are chosen of opposite colors.

Let $A=\left\{r_{1}, b_{2}\right\}$ or $A=\left\{b_{1}, r_{2}\right\}$ arbitrarily, containing two endpoints of the path. At every step, recompute the top and bottom bridges of $S \backslash A$, and append either the red or the blue vertex of each bridge to $A$ such that the appended edges cross the separating line $\ell$. In the last step, the convex hull of $S \backslash A$ is a red-blue segment that merges the two sub-paths. The two new edges added simultaneously at each step cannot cross each other; and they cannot cross previous edges, since they are separated from them by the convex hull of $S \backslash A$. Finally, they cannot extend the two sub-paths by the same point either, because $|S|$ is even.

The next lemma follows from [13, Lemma 2.1]; we will only need its corollary, Lemma 11.

Lemma 10 ([13]) Let $P=\left(p_{1}, p_{2}, \ldots, p_{n}\right)$ be a simple polygon (with the convention that $\left.p_{n+1}=p_{1}\right)$ and $q$ be a point in the exterior of the convex hull of $P$, where 
$V(P) \cup\{q\}$ is in general position. Then $q$ sees one edge $p_{i} p_{i+1}$ of $P$. Such an edge can be found in $O(n)$ time.

Lemma 11 Let $P=\left(p_{1}, p_{2}, \ldots, p_{n}\right)$ be a simple polygon (with the convention that $\left.p_{n+1}=p_{1}\right)$ and $q$ be a point in the exterior of the convex hull of $P$, where $V(P) \cup\{q\}$ is in general position. Then the polygonal cycle $P$ can be extended to include $q$ so that $P \cup\{q\}$ is still a simple polygon. More precisely, there exists $i \in[n]$, so that $Q=\left(p_{1}, \ldots, p_{i}, q, p_{i+1}, \ldots, p_{n}\right)$ is a simple polygon. Moreover, $L(Q)>L(P)$. The extension can be computed in $O(n)$ time.

Proof By Lemma 10, $q$ sees one edge $p_{i} p_{i+1}$ of $P$. Replacing this edge of $P$ by the two edges $p_{i} q$ and $q p_{i+1}$ results in a simple polygon $Q=\left(p_{1}, \ldots, p_{i}, q, p_{i+1}, \ldots\right.$, $\left.p_{n}\right)$. By the triangle inequality, $L(Q)>L(P)$. The extension can be computed in $O(n)$ time, as determined by the time needed to find a visible edge.

Note that the condition in the lemma that $q$ lies in the exterior of the convex hull of $P$, is indeed necessary. Otherwise one cannot guarantee that $q$ sees an edge of $P$.

(i) Our first algorithm. We are now ready to present our Algorithm A4. Assume first for simplicity that $m$ is even. An easy modification of the algorithm, explained below, is used if $m$ is odd.

Algorithm A4 STEP 1. For all non-equivalent bisections of $S^{\prime \prime}$ (i.e., for all balanced bipartitions of $S^{\prime \prime}$ ): 1 . Compute a non-crossing alternating path $A$ of $S^{\prime \prime}$ by using the two-endpoint path construction algorithm (Lemma 9). 2. Extend $A$ to a cycle by connecting its endpoints to (one or two) convex hull vertices. 3. Further extend this cycle to include the remaining hull vertices, by repeated invocation of Lemma 11.

STEP 2. Output the longest such cycle (containing all points of $S$ ).

Observe that after STEP 1.1, the two endpoints of the path are vertices of $\operatorname{conv}\left(S^{\prime \prime}\right)$, hence they can be connected to hull vertices in $S^{\prime}$ to make a cycle. If $m$ is odd, then there is a point $q \in S^{\prime \prime}$ on the line $\ell$. Use the two-endpoint path construction algorithm for $S^{\prime \prime} \backslash\{q\}$, and the same bisecting line $\ell$. If $q$ is in the interior of $\operatorname{conv}\left(S^{\prime \prime} \backslash\{q\}\right)$, then extend the path with point $q$, using Lemma 2. Otherwise, $q$ sees the top or bottom bridge of $\operatorname{conv}\left(S^{\prime \prime} \backslash\{q\}\right)$, so the path can be extended by connecting $q$ to the endpoint visible to $q$. The two endpoints of the extended path are on $\operatorname{conv}\left(S^{\prime \prime}\right)$, hence they can be connected to hull vertices to make a cycle, as in the case of even $m$.

See Fig. 12 for an illustration of the algorithm on a small example. We now justify its correctness and estimate the length of the output cycle. Assume first that $m$ is even. For a given $\alpha$, let $A_{\alpha}$ be a non-crossing alternating path with respect to a balanced bipartition of $S^{\prime \prime}$ induced by the $y$-axis of $\Gamma_{\alpha}$, as computed by the algorithm. Assume that $A_{\alpha}=\left(p_{i_{1}}, p_{i_{2}}, \ldots, p_{i_{m}}\right)$. By projecting on the $x$-axis of $\Gamma_{\alpha}$, we get

$$
\begin{aligned}
L\left(A_{\alpha}\right) & \geq\left|x_{i_{1}}\right|+2\left|x_{i_{2}}\right|+\cdots+2\left|x_{i_{m-1}}\right|+\left|x_{i_{m}}\right| \\
& =2 \sum_{i=1}^{n}\left|x_{i}\right|-\left|x_{i_{1}}\right|-\left|x_{i_{m}}\right|-2\left(\left|x_{j_{1}}\right|+\cdots+\left|x_{j_{h}}\right|\right)
\end{aligned}
$$



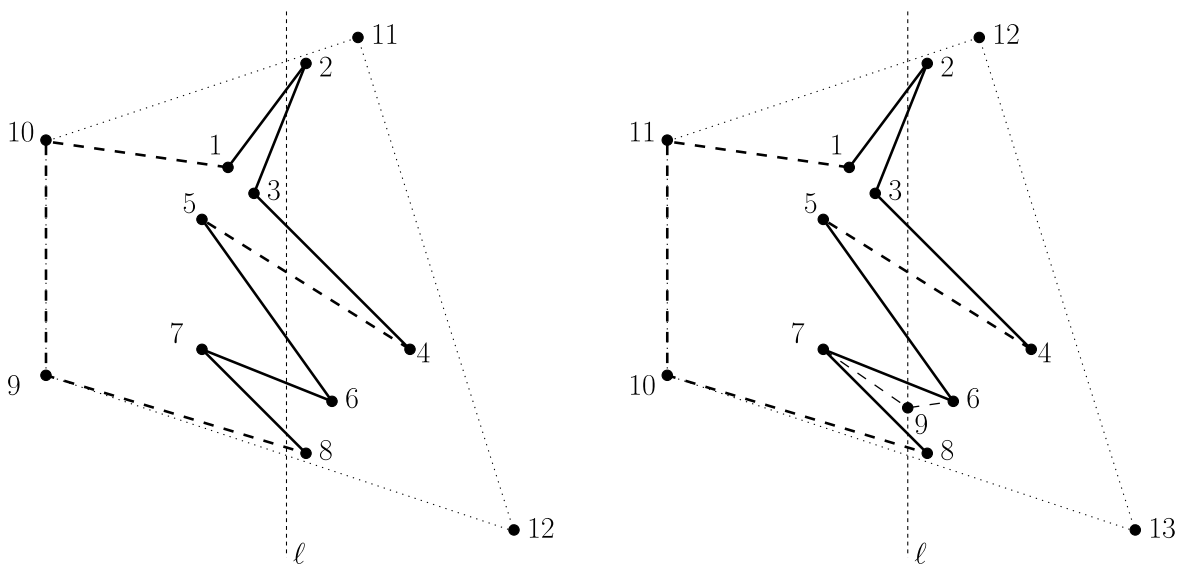

Fig. 12 Left: an alternating path $(1,2, \ldots, 8)$ obtained by using the two-endpoint path construction algorithm (Lemma 9) for the case of even $m$. The path is extended to a cycle by connecting its endpoints to convex hull vertices (here 9 and 10$)$. The cycle $(1,2, \ldots, 10)$ will be subsequently extended to include the remaining hull vertices (here 11 and 12) using Lemma 11. Right: an alternating path $(1,2, \ldots, 8)$ obtained in the same way, and then extended to pass through vertex 9 (Lemma 2); the resulting path is $(1,2,3,4,5,6,9,7,8)$. The path is extended to a Hamiltonian cycle in the same way (Lemma 11)

$$
\begin{aligned}
& =\sum_{j=1}^{n}\left(\left|x_{j}\right|+\left|x_{j+1}\right|\right)-\left|x_{i_{1}}\right|-\left|x_{i_{m}}\right|-2\left(\left|x_{j_{1}}\right|+\cdots+\left|x_{j_{h}}\right|\right) \\
& \geq \sum_{j=1}^{n}\left(\left|x_{j}\right|+\left|x_{j+1}\right|\right)-W_{\alpha}-(2 h-2) W_{\alpha} \\
& \geq \sum_{j=1}^{n}\left|p_{j} p_{j+1}\right|\left|\cos \left(\beta_{j, j+1}-\alpha\right)\right|-(2 h-1) W_{\alpha} .
\end{aligned}
$$

In the above chain of inequalities we have used the facts that $m$ is even, thus $p_{i_{1}}$ and $p_{i_{m}}$ lie on opposite sides of $\ell$, and that at least two convex hull vertices are also separated by $\ell$. The inequality is maintained for $m$ odd, as in the proof of Theorem 1 .

Let $A=\left(p_{i_{1}}, p_{i_{2}}, \ldots, p_{i_{m}}\right)$ be the longest of the $O(k)$ Hamiltonian non-crossing paths $A_{\alpha_{i}}$ over all $O(k)$ balanced bipartitions of $S^{\prime \prime}$. (In estimating the length of the output cycle, it is enough to analyze the augmentation of $A$ to a Hamiltonian cycle, rather than of each path $A_{\alpha}$.) Let $u$ and $v$ be (hull) vertices in $S^{\prime}$ that can be connected with the two endpoints $p_{i_{1}}$ and $p_{i_{m}}$ of $A$ without crossings any edge of $A$ ( $u$ and $v$ may be forced to coincide). Let $P$ be the simple cycle (polygon) obtained by adding to $A$ the points on one of the two convex hull chains connecting $u$ and $v$. In the last phase, $P$ is further extended by adding the remaining hull vertices (on the 2nd hull chain connecting $u$ and $v$ ) one by one by Lemma 11 . Note that the length of the cycle increases with each point addition. The total time for all additions is trivially bounded by $O\left(n^{2}\right)$. The resulting polygonal cycle $Q$ is non-crossing and spans all points in $S$, 
therefore making a Hamiltonian cycle of $S$. Its length is bounded as follows.

$$
L(Q) \geq L(A) \geq \sum_{j=1}^{n}\left|p_{j} p_{j+1}\right|\left|\cos \left(\beta_{j, j+1}-\alpha\right)\right|-(2 h-1) W_{\alpha},
$$

and by integration over the $\alpha$-interval $[0, \pi]$,

$$
L(Q) \geq \frac{2}{\pi} L\left(Q_{\mathrm{OPT}}\right)-(2 h-1) \frac{P}{\pi} .
$$

If the point set satisfies the condition $(2 h-1) \frac{P}{\pi} \leq \delta L\left(Q_{\mathrm{OPT}}\right)$, then

$$
L(Q) \geq \frac{2}{\pi} L\left(Q_{\mathrm{OPT}}\right)-\delta L\left(Q_{\mathrm{OPT}}\right)=\left(\frac{2}{\pi}-\delta\right) L\left(Q_{\mathrm{OPT}}\right) .
$$

This concludes the proof of part (i) of Theorem 3 .

(ii) Our second algorithm. One can reduce the term $(2 h-1) \frac{P}{\pi}$ in Inequality (7) to $(h+1) \frac{P}{\pi}$ at the cost of increasing the running time to $O\left(n^{3} \log n\right)$. We replace the balanced partitions of $S^{\prime \prime}$ by two unbalanced partitions of $S$. Recall that a line has direction $\alpha \in[0, \pi)$ if it makes a counterclockwise angle of $\alpha$ with the $x$-axis. Let $S$ be a set of $n$ points in the plane in general position. A direction $\alpha \in[0, \pi)$ is general if the supporting line of no two points in $S$ has direction $\alpha$. The lines of a general direction $\alpha$ partition $S$ in $2 n+1$ different ways (into subsets lying on the left, on the right, and on the line). Two directions, $\alpha$ and $\beta$, are equivalent if they induce the same $2 n+1$ partitions of $S$. There are exactly $\left(\begin{array}{c}n \\ 2\end{array}\right)$ non-equivalent directions.

Recall that $S=S^{\prime} \cup S^{\prime \prime}$, where $S^{\prime}$ is the set of convex hull vertices and $S^{\prime \prime}$ is the set of interior points. Fix a general direction $\alpha \in(0, \pi)$. A line $\ell$ of direction $\alpha$ is left-balanced if the number of points of $S$ in the left open halfplane equals the number of points of $S^{\prime \prime}$ in the right open halfplane. It is easy to see that all leftbalanced lines are equivalent. Indeed if we continuously move a line $\ell$ of direction $\alpha$ from left to right, then the number of points of $S$ in the left open halfplane increases every time $\ell$ passes through a point of $S$, and the number of points of $S^{\prime \prime}$ in the right open halfplane monotonically decreases. Therefore, there is equality for exactly one equivalence class of lines of direction $\alpha$. Denote by $S_{\alpha}^{1}$ the set of all points of $S^{\prime \prime}$ and the points in $S^{\prime}$ lying in the left closed halfplane of a left-balanced line. Clearly, every left-balanced line is a bisecting line for $S_{\alpha}^{1}$.

Similarly, a line $\ell$ of direction $\alpha$ is right-balanced if the number of points of $S$ in the right open halfplane equals the number of points of $S^{\prime \prime}$ in the left open halfplane. Denote by $S_{\alpha}^{2}$ the set of all points of $S^{\prime \prime}$ and the points in $S^{\prime}$ lying in the right closed halfplane of a right-balanced line. Note that a left-balanced line lies to the left of a right-balanced line, in particular, they are separated by the bisecting line of $S$ of direction $\alpha$. We now present our Algorithm A4'.

Algorithm A4' STEP 1. For all non-equivalent general directions $\alpha$, compute a leftbalanced line $\ell_{\alpha}^{1}$, a right-balanced line $\ell_{\alpha}^{2}$, and the two sets $S_{\alpha}^{1}$ and $S_{\alpha}^{2}$. For $i=1,2$ do: 1 . If $\left|S_{\alpha}^{i}\right|$ is even, compute a non-crossing alternating path $A$ for $S_{\alpha}^{i}$ using the twoendpoint path construction algorithm (in Lemma 9). 2. If $\left|S_{\alpha}^{i}\right|$ is odd, then $\ell_{\alpha}^{i}$ passes 

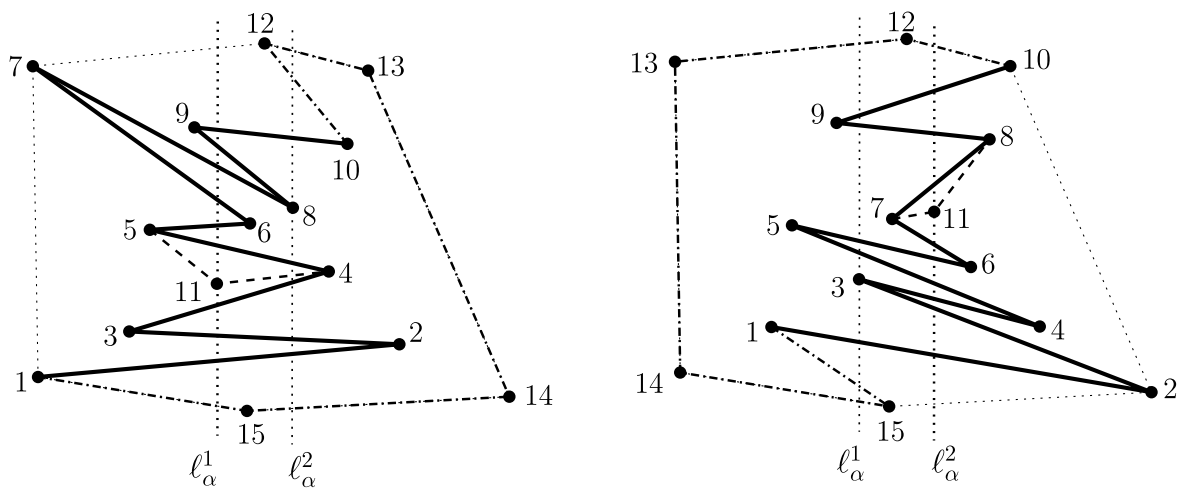

Fig. 13 Left: an alternating path 1,2, ., 10 constructed by the two-endpoint path construction algorithm (Lemma 9) for the set bisected by $\ell_{\alpha}^{1}$. It is augmented to pass through point 11 incident to $\ell_{\alpha}^{1}$, and then to a Hamiltonian cycle along the remaining convex hull vertices $12,13,14,15$. Right: an alternating path, an augmented path, and a Hamiltonian cycle analogously computed for line $\ell_{\alpha}^{2}$

through some point $q \in S_{\alpha}^{i}$. Compute a non-crossing alternating path for $S_{\alpha}^{i} \backslash\{q\}$ with the two-endpoint path construction algorithm, and then extend it to include $q$ as follows: if $q$ is in the interior of $\operatorname{conv}\left(S_{\alpha}^{i}\right)$, then use Lemma 2 to augment the alternating path with $q$; otherwise connect $q$ to the endpoint of the path lying on the upper or lower bridge of $\operatorname{conv}\left(S_{\alpha}^{i}\right)$, whichever is visible from $q$. 3. Complete the path $A$ on $S_{\alpha}^{i}$ to a cycle through the remaining convex hull vertices in $S \backslash S_{\alpha}^{i}$.

STEP 2. Output the longest such cycle (containing all points of $S$ ).

We now analyze this algorithm. For a given $\alpha \in(0, \pi)$, let $A_{\alpha}^{1}$ and $A_{\alpha}^{2}$ be the two non-crossing paths computed in STEP 1(1-2) of Algorithm A4' for the lines $\ell_{\alpha}^{1}$ and $\ell_{\alpha}^{2}$, respectively. The path $A_{\alpha}^{1}$ is a Hamiltonian path on $S_{\alpha}^{1}$ such that one endpoint is an endpoint of the top bridge of $\operatorname{conv}\left(S_{\alpha}^{1}\right)$, and the other endpoint is an endpoint of the bottom bridge of $\operatorname{conv}\left(S_{\alpha}^{1}\right)$ with respect to $\ell_{\alpha}^{1}$. Since $\operatorname{conv}\left(S_{\alpha}^{1}\right) \subset \operatorname{conv}(S)$, the endpoints of the top (resp., bottom) bridge of $\operatorname{conv}(S)$ and the top (resp., bottom) bridge of $\operatorname{conv}\left(S_{\alpha}^{1}\right)$ are in convex position. Hence, the edges that connect the endpoints of $A_{\alpha}^{1}$ to the top and bottom vertices of $\operatorname{conv}\left(S \backslash S_{\alpha}^{1}\right)$ lie outside of $\operatorname{conv}\left(S_{\alpha}^{1}\right)$, and so they do not cross any edge of $A_{\alpha}^{1}$, which lies inside conv $\left(S_{\alpha}^{1}\right)$. This shows that in STEP 1(3) of Algorithm $\mathrm{A}^{\prime}$, the path $A_{\alpha}^{1}$ is completed to a non-crossing Hamiltonian cycle. Analogously, the path $A_{\alpha}^{2}$ is also completed to a non-crossing Hamiltonian cycle.

There are $\left(\begin{array}{l}n \\ 2\end{array}\right)=O\left(n^{2}\right)$ non-equivalent general directions $\alpha \in(0, \pi)$. In each direction $\alpha$, we can compute $\ell_{\alpha}^{1}, \ell_{\alpha}^{2}, S_{\alpha}^{1}$ and $S_{\alpha}^{2}$ in $O(n \log n)$ time by a sweep line algorithm. By Lemma 1, we can compute the alternating paths $A_{\alpha}^{1}$ and $A_{\alpha}^{2}$ in $O(n \log n)$ time. Consequently the total running time of $\mathrm{A} 4^{\prime}$ is $O\left(n^{3} \log n\right)$.

Fix a general direction $\alpha \in(0, \pi)$, a left-balanced line $\ell_{\alpha}^{1}$, and a right-balanced line $\ell_{\alpha}^{2}$. Assume that $S_{\alpha}^{1}=\left\{p_{i}: i \in I\right\}$ and $S_{\alpha}^{2}=\left\{p_{j}: j \in J\right\}$. Denote by $x_{1}(p)$ and $x_{2}(p)$ the distance of a point $p$ from the lines $\ell_{\alpha}^{1}$ and $\ell_{\alpha}^{2}$, respectively. A point $p \in S^{\prime}$ in the closed slab between $\ell_{\alpha}^{1}$ and $\ell_{\alpha}^{2}$ is incident to neither $A_{\alpha}^{1}$ nor $A_{\alpha}^{2}$; for such a point we have $x_{1}(p)+x_{2}(p) \leq W_{\alpha}$. Every point $p \in S^{\prime}$ to the left of $\ell_{\alpha}^{1}$ or to the right of $\ell_{\alpha}^{2}$ is incident to exactly one of $A_{\alpha}^{1}$ and $A_{\alpha}^{2}$, and for such a point we have 
$\max \left\{x_{1}(p), x_{2}(p)\right\} \leq W_{\alpha}$. This implies that $\sum_{i \notin I} x_{1}\left(p_{i}\right)+\sum_{j \notin J} x_{2}\left(p_{j}\right) \leq\left|S^{\prime}\right| W_{\alpha}=$ $h W_{\alpha}$. Similarly to (6), we obtain

$$
\begin{aligned}
& L\left(A_{\alpha}^{1}\right)+L\left(A_{\alpha}^{2}\right) \\
& \quad \geq\left(2 \sum_{i \in I} x_{1}\left(p_{i}\right)-W_{\alpha}\right)+\left(2 \sum_{j \in J} x_{2}\left(p_{j}\right)-W_{\alpha}\right) \\
& =2\left(\sum_{i=1}^{n} x_{1}\left(p_{i}\right)-\sum_{i \notin I} x_{1}\left(p_{i}\right)\right)+2\left(\sum_{j=1}^{n} x_{2}\left(p_{j}\right)-\sum_{j \notin J} x_{2}\left(p_{j}\right)\right)-2 W_{\alpha} \\
& =2 \sum_{i=1}^{n} x_{1}\left(p_{i}\right)+2 \sum_{i=1}^{n} x_{2}\left(p_{i}\right)-2\left(\sum_{i \notin I} x_{1}\left(p_{i}\right)+\sum_{j \notin J} x_{2}\left(p_{j}\right)\right)-2 W_{\alpha} \\
& \geq 2 \sum_{i=1}^{n} x_{1}\left(p_{i}\right)+2 \sum_{i=1}^{n} x_{2}\left(p_{i}\right)-2 h W_{\alpha}-2 W_{\alpha} \\
& =\sum_{i=1}^{n}\left[x_{1}\left(p_{i}\right)+x_{1}\left(p_{i+1}\right)\right]+\sum_{i=1}^{n}\left[x_{2}\left(p_{i}\right)+x_{2}\left(p_{i+1}\right)\right]-(2 h+2) W_{\alpha} \\
& \geq 2 \sum_{i=1}^{n}\left|p_{i} p_{i+1}\right|\left|\cos \left(\beta_{i, i+1}-\alpha\right)\right|-(2 h+2) W_{\alpha} .
\end{aligned}
$$

Consequently,

$$
\max \left\{L\left(A_{\alpha}^{1}\right), L\left(A_{\alpha}^{2}\right)\right\} \geq \sum_{i=1}^{n}\left|p_{i} p_{i+1}\right|\left|\cos \left(\beta_{i, i+1}-\alpha\right)\right|-(h+1) W_{\alpha} .
$$

By integration over the $\alpha$-interval $[0, \pi]$, we obtain the desired bound

$$
L(Q) \geq \frac{2}{\pi} L\left(Q_{\mathrm{OPT}}\right)-\frac{h+1}{\pi} P .
$$

The proof of part (iii) is analogous to that of part (iii) of Theorem 1. This concludes the proof of Theorem 3 .

We now discuss an example illustrating part (i). Denote by $\mathrm{E}[U]$ the expected value of a random variable $U$. According to a result of Rényi and Sulanke (1963), if $n$ points are chosen uniformly and independently at random from a plane convex $r$-gon $(r$ fixed), then $\mathrm{E}[h]=O(\log n)$, where the hidden constant depends on $r[19$, pp. 151]. According to a another result of Raynaud (1970), if $n$ points are chosen uniformly and independently at random in a circle, then $\mathrm{E}[h]=O\left(n^{1 / 3}\right)[19$, pp. 151]. Hence for a set of $n$ random points uniformly selected in a convex polygon or a circle, $h=O\left(n^{1 / 3}\right)$ with probability close to 1 . Let the selection region have unit diameter. Then also with high probability, we have $L\left(M_{\mathrm{OPT}}\right)=\Omega(n)$, thus also $L\left(Q_{\mathrm{OPT}}\right)=\Omega(n)$. Since $P \leq \pi$, we have $(2 h-1) \frac{P}{\pi} \leq \delta L\left(Q_{\mathrm{OPT}}\right)$ with high probability, for a very small $\delta=O\left(1 / n^{2 / 3}\right)$. Thus by inequality (7), the cycle returned by 
A4 is a $(2 / \pi-o(1))$ approximation of the optimal path for random point instances as described above.

\section{Concluding Remarks}

It is generally believed that the number of halving lines of a point set is $O\left(n^{1+\varepsilon}\right)$, for any $\varepsilon>0[9,11,20]$, so it is also very likely that the running time of our algorithm for Hamiltonian paths ( $n$ even) is also $O\left(n^{2+\varepsilon}\right)$, for any $\varepsilon>0$.

Similarly to [3], our approximation factors are in terms of $L\left(H_{\mathrm{OPT}}\right)$ and $L\left(T_{\mathrm{OPT}}\right)$ respectively, rather than their non-crossing counterparts. In other words, the trivial bounds $L\left(H_{\mathrm{OPT}}^{*}\right) \leq L\left(H_{\mathrm{OPT}}\right)$, and $L\left(T_{\mathrm{OPT}}^{*}\right) \leq L\left(T_{\mathrm{OPT}}\right)$ are used in the proofs. It is not clear if one can use the lengths of the non-crossing optimal structures for improving the approximation ratios. The situation is similar for the Hamiltonian cycle problem. It is worth studying whether the lower bound estimate on the length of the cycle returned by our Algorithm A4 may be used to derive an approximation ratio that holds for all instances, similarly to the Hamiltonian path problem. A more precise analysis of the approximation ratio of our Algorithm A3 for non-crossing spanning tree construction remains as another open problem. We believe that the approximation ratio of $\mathrm{A} 3$ is substantially better than $1 / 2$.

Acknowledgements The authors thank Gruia Călinescu for valuable remarks and for many interesting conversations on the topic.

\section{References}

1. Abellanas, M., Garcia, J., Hernández, G., Noy, M., Ramos, P.: Bipartite embeddings of trees in the plane. Discrete Appl. Math. 93, 141-148 (1999)

2. Aichholzer, O., Cabello, S., Fabila-Monroy, R., Flores-Peñaloza, D., Hackl, T., Huemer, C., Hurtado, F., Wood, D.R.: Edge-removal and non-crossing configurations in geometric graphs. Discrete Math. Theor. Comput. Sci. 12(1), 75-86 (2010)

3. Alon, N., Rajagopalan, S., Suri, S.: Long non-crossing configurations in the plane. Fund. Inf. 22, 385394 (1995). Also in Proc. of the 9-th Annual Symposium on Computational Geometry, pp. 257-263. ACM Press (1993)

4. Asano, T., Ghosh, S., Shermer, T.: Visibility in the plane. In: Sack, J.-R., Urrutia, J. (eds.) Handbook of Computational Geometry, pp. 829-876. Elsevier, Amsterdam (2000)

5. Bern, M., Eppstein, D.: Approximation algorithms for geometric problems. In: Hochbaum, D.S. (ed.) Approximation Algorithms for NP-hard Problems, pp. 296-345. PWS, Boston (1997)

6. Černý, J., Dvorák, Z., Jelínek, V., Kára, J.: Noncrossing Hamiltonian paths in geometric graphs. Discrete Appl. Math. 155, 1096-1105 (2007)

7. Dey, T.K.: Improved bounds on planar $k$-sets and related problems. Discrete Comput. Geom. 19, 373-382 (1998)

8. Edelsbrunner, H.: Algorithms in Combinatorial Geometry. Springer, Heidelberg (1987)

9. Edelsbrunner, H., Welzl, E.: On the number of separations of a finite set in the plane. J. Comb. Theory Ser. A 38, 15-29 (1985)

10. Eppstein, D.: Spanning trees and spanners. In: Sack, J.-R., Urrutia, J. (eds.) Handbook of Computational Geometry, pp. 425-461. Elsevier, Amsterdam (2000)

11. Erdős, P., Lovász, L., Simmons, A., Straus, E.: Dissection graphs of planar point sets. In: Srivastava, J.N. (ed.) A Survey of Combinatorial Theory, pp. 139-154. North-Holland, Amsterdam (1973)

12. Fekete, S.P.: Simplicity and hardness of the maximum traveling salesman problem under geometric distances. In: Proceedings of the 10-th ACM-SIAM Symposium on Discrete Algorithms, January 1999 (SODA '99), pp. 337-345 
13. Hurtado, F., Kano, M., Rappaport, D., Tóth, Cs.D.: Encompassing colored planar straight line graphs. Comput. Geom., Theory Appl. 39(1), 14-23 (2008)

14. Károlyi, G., Pach, J., Tóth, G.: Ramsey-type results for geometric graphs. I. Discrete Comput. Geom. 18, 247-255 (1997)

15. Károlyi, G., Pach, J., Tóth, G., Valtr, P.: Ramsey-type results for geometric graphs. II. Discrete Comput. Geom. 20, 375-388 (1998)

16. Lovász, L.: On the number of halving lines. Ann. Univ. Sci. Budapest, Eötvös, Sec. Math. 14, 107-108 (1971)

17. Mitchell, J.S.B.: Geometric shortest paths and network optimization. In: Sack, J.-R., Urrutia, J. (eds.) Handbook of Computational Geometry, pp. 633-701. Elsevier, Amsterdam (2000)

18. O'Rourke, J.: Visibility. In: Goodman, J., O'Rourke, J. (eds.) Handbook of Discrete and Computational Geometry, 2nd edn., pp. 643-663. Chapman \& Hall, London (2004)

19. Preparata, F., Shamos, M.: Computational Geometry: An Introduction. Springer, New York (1985)

20. Tóth, G.: Point sets with many $k$-sets. Discrete Comput. Geom. 26, 187-194 (2001)

21. Yaglom, I.M., Boltyanskiǔ, V.G.: Convex Figures. Holt, Rinehart and Winston, New York (1961) 